\title{
FINITE TEST SETS AND $P$-MATRICES
}

\author{
MICHAEL M. KOSTREVA
}

\begin{abstract}
The class of matrices with all principal minors positive, known as $\boldsymbol{P}$-matrices, has been characterized by Murty and Tamir using a finite set of test vectors for the linear complementarity problem. This paper refines their characterizations by deriving a set of test vectors which has lower cardinality and vectors which are more easily tested.
\end{abstract}

Consider the following problem, known as the linear complementarity problem:

Given a vector $q \in R^{n}$ and an $n$ by $n$ matrix $M$, find a vector $x \in R^{n}$ satisfying

$$
y=q+M x, \quad y \geqslant 0, \quad x \geqslant 0, \quad y^{t} x=0 .
$$

For convenience, let the abbreviation LCP represent the linear complementarity problem. A matrix $\boldsymbol{M}$ is a $\boldsymbol{P}$-matrix if all principal minors of $\boldsymbol{M}$ are positive. If the LCP with data $(q, M)$ has a solution for each $q \in R^{n}, M$ is called a $Q$-matrix. The superscript $t$ denotes matric transposition. If $A$ is any matrix, $A_{{ }_{\bullet} j}$ denotes column $j$. $I$ is the identity matrix and $e$ is a vector of l's.

The LCP is a mathematical structure which has inspired great interest in the past fifteen years. The LCP is mostly linear, with the exception of the single nonlinear constraint $y^{t} x=0$. With its "minimal" amount of nonlinearity, the LCP is appealing yet mathematically tractable. Many related mathematical models may be viewed in the general framework of the LCP. Some examples are linear and quadratic programming, the Nash equilibrium of noncooperative games, Leontief input-output models, free boundary problems for differential operators, economic equilibria and partitioning the finite dimensional Euclidean space $R^{n}$. Hence, we are motivated to study questions concerning the existence and uniqueness of solutions for this important problem.

A key result in complementarity theory is that LCP has a unique solution for each $q \in R^{n}$ if and only if $M$ is a $P$-matrix [1, 2]. Refining this characterization [3], Murty introduced the notion of a finite test set for LCP: if the LCP has a unique solution for each vector $q$ in the collection

$$
\Gamma=\left\{I_{\cdot 1}, \ldots, I_{\cdot n},-I_{\cdot 1}, \ldots,-I_{\cdot n}, M_{\cdot 1}, \ldots, M_{\cdot n},-M_{\cdot 1}, \ldots,-M_{\cdot n}, e\right\},
$$

then $M$ is a $P$-matrix and hence the LCP has a unique solution for each $q \in R^{n}$. Note that $\Gamma$ has $(4 n+1)$ select elements.

Tamir [4] studied the test set $\Gamma_{1}$ where $\Gamma_{1}=\left\{I_{\cdot 1}, \ldots, I_{\cdot n}, M_{\cdot 1}, \ldots, M_{\cdot n}\right.$, $\left.-M_{\cdot 1}, \ldots,-M_{\cdot n}, e\right\}$ of cardinality $(3 n+1)$. He found that $\Gamma_{1}$ was sufficient for testing whether or not $M$ is a $P$-matrix. If an efficient algorithm can be found for

Received by the editors September 16, 1980; presented November 10, 1980 to the Joint National Meeting of the Operations Research Society of America and the Institute of Management Sciences.

1980 Mathematics Subject Classification. Primary 90C33, 65F40, 15A48; Secondary 15A15, 90 C20.

(c) 1982 American Mathematical Society 0002-9939/82/0000-0024/\$01.50 
testing the uniqueness of the solution to LCP for any $q$, then testing existence (with some constructive algorithm) and uniqueness of solutions on $\Gamma$ or $\Gamma_{1}$ would likely be more efficient than computation of the $2^{n}-1$ principal subdeterminants of the matrix $M$.

The main result of this paper is a further refinement in the form of a finite test set for LCP.

TheOREM. The $n$ by $n$ matrix $M$ is a $P$-matrix if and only if LCP has a unique solution for each $q$ in the set $\Gamma_{2}$ where $\Gamma_{2}$ has cardinality $(2 n+2)$ and $\Gamma_{2}=\left\{I_{\cdot 1}, \ldots, I_{\cdot n},-M_{\cdot 1}, \ldots,-M_{\cdot n}, e, 0\right\}$.

Proof. If LCP has a unique solution for $q \in\{e, 0\}$, then Lemke's Algorithm [5] proves that $M$ is a $Q$-matrix: there can be no secondary ray. Following Garcia [6], $M \in Q$, since Lemke's Algorithm with covering vector $d=e$ must terminate in a solution for each $q \in R^{n}$. Next observe as in [3] that if $M$ is a $Q$-matrix and LCP has a unique solution corresponding to each vector $q \in\left\{I_{\cdot 1}, \ldots, I_{\bullet n},-M_{\cdot 1}, \ldots\right.$, $\left.-M_{\cdot n}\right\}$, then $M$ is a $P$-matrix. Taking the union of the two sets yields the forward implication.

If $M$ is a $P$-matrix, then existence and uniqueness of solutions to the LCP for all $q \in R^{n}$ assures the same property on $\Gamma_{2} \subseteq R^{n}$.

The test set $\Gamma_{2}$ refines the earlier sets $\Gamma$ and $\Gamma_{1}$ in several ways. Cardinality is lower which aids the checking. The set $X=\left\{0, \ldots, 0, I_{\cdot 1}, \ldots, I_{\cdot n}, 0,0\right\}$ contains explicit solutions to the LCP for $q$ in $\Gamma_{2}=\left\{I_{\cdot 1}, \ldots, I_{\cdot n},-M_{\cdot 1}, \ldots,-M_{\cdot n}, e, 0\right\}$. Thus only uniqueness need be checked on $\Gamma_{2}$, unlike $\Gamma$ and $\Gamma_{1}$ where both existence and uniqueness need to be checked.

\section{REFERENCES}

1. H. Samelson, R. Thrall and O. Wesler, A partition theorem for Euclidean $n$-space, Proc. Amer. Math. Soc. 9 (1958), 805-807.

2. K. G. Murty, On the number of solutions to the complementarity problem and spanning properties of complementary cones, Linear Algebra and Appl. 5 (1972), 65-108.

3. __ On a characterization of P-matrices, SIAM J. Appl. Math. 20 (1971), 378-383.

4. A. Tamir, On a characterization of P-matrices, Math. Programming 4 (1973), 110-112.

5. C. E. Lemke, Bimatrix equilibrium points and mathematical programming, Management Sci. 11 (1965), 681-689.

6. C. B. Garcia, Some classes of matrices in linear complementarity theory, Math. Programming 5 (1973), 299-310.

Mathematics Department, General Motors Research Laboratories, Warren, Michigan 48090 